\title{
AVALIAÇÃO DA CASCA DE PASSIFLORA EDULIS COMO FONTE DE FLAVONÓIDES
}

\author{
J. R. SOUSA ${ }^{1}$, M. Y. V. FARIAS ${ }^{1}$, C. M. G. F. LEMOS ${ }^{1}$, J. A. SILVA², M. C. M. SOUZA ${ }^{3}$ e \\ L. R. B. GONÇALVES ${ }^{1}$ \\ ${ }^{1}$ Universidade Federal do Ceará, Departamento de Engenharia Química \\ ${ }^{2}$ Universidade Federal de Sergipe, Departamento de Farmácia \\ ${ }^{3}$ Universidade da Integração Internacional da Lusofonia Afro-Brasileira - Instituto de \\ Engenharias e Desenvolvimento Sustentável \\ E-mail para contato: julianarabello@yahoo.com.br
}

\begin{abstract}
RESUMO - Flavonóides possuem grande espectro de ação biológica, tais como, atividade antioxidante, anti-inflamatória e antitumoral. Entretanto, sua utilização em formulações industriais é limitada devido à baixa solubilidade em meios lipofílicos. A esterificação por lipases tem sido utilizada para melhorar as propriedades dos flavonoides. Desta forma, avaliou-se a espécie Passiflora edulis (maracujá) como matéria-prima para obtenção de flavonoides que serão submetidos à esterificação utilizando lipase de Candida antarctica tipo B (CALB) imobilizada em nanoparticulas magnéticas e cujos resultados serão comparados com CALB imobilizada em resina acrílica comercial. Os extratos etanólicos foram obtidos por extração sólido-líquido da casca do maracujá seca que, após evaporação do solvente, foram liofilizados e armazenados a $4{ }^{\circ} \mathrm{C}$. Determinouse o teor de flavonoides em HPLC empregando detector UV/PDA. Obteve-se $35 \mathrm{mg} / \mathrm{L}$ de flavonoides nos extratos, indicando o potencial do maracujá como fonte destes antioxidantes.
\end{abstract}

\section{INTRODUÇÃO}

Flavonóides são compostos fenólicos naturais que ocorrem em uma grande variedade de espécies vegetais e exibem inúmeros efeitos benéficos para a saúde humana. Estas substâncias apresentam grande poder antioxidante além de possuírem efeito vasodilatador, antiinflamatório, anticancerígeno, antiviral e antibacteriano (Bendini et al., 2006). Grande quantidade desses flavonoides podem ser encontrados na espécie Passiflora edulis (Passifloraceae), popularmente conhecida como maracujá amarelo (Rudnicki et al., 2007). Cascas dos frutos deste vegetal são subprodutos da indústria alimentícia e, portanto são considerados resíduos industriais. Estas indústrias geram uma grande quantidade desses resíduos, pois juntamente com suas sementes representam cerca de $70 \%$ do peso do fruto (Oliveira et al., 2002). O Brasil é um dos principais produtores mundiais de maracujá, e a produção de frutos, em 2009, concentrou-se nos estados da Bahia, Ceará, Sergipe, Espírito Santo e Minas Gerais, representando cerca de $80 \%$ da produção nacional. O Ceará é o segundo maior produtor de maracujá do Brasil, representando cerca de $18 \%$ da produção nacional (EMBRAPA).

Apesar de todas as ações benéficas dos flavonoides, sua baixa hidrofobicidade prejudica a sua ação farmacológica devido a reduzida capacidade em atravessar membranas biológicas e consequente redução na distribuição tecidual em organismos vivos. Além disso, a dificuldade 
de incorporação desses antioxidantes em algumas formulações farmacêuticas e produtos alimentícios também é um problema por causa de sua baixa lipossolubilidade (Passicos et al., 2004; Wang et al., 2010).

Os flavonoides que se apresentam na forma glicosilada possuem em sua estrutura hidroxilas primárias glicosídicas que constituem pontos suscetíveis de funcionalização por lipases. Lipases são classificadas como hidrolases (triacilglicerolacil-hidrolases, EC 3.1.1.3), mas são capazes de catalisar reações reversas, como esterificação, transesterificação e lactonização em meio não aquoso (Sharma e Kanwar, 2014; Arcos et al., 2001). A esterificação seletiva da hidroxila primária glicosídica de flavonoides catalisada por lipase confere à estes antioxidantes aumento da lipofilidade acarretando um aumento em sua afinidade por membranas biológicas e, desta forma, otimizando a ação farmacológica desejada.

A espécie $P$. edulis apresenta principalmente em sua constituição os flavonóides: 2lucenina, 2-vicenina, isoorientina, isovitexina, luteolina-6-C-chinovosídeo e luteolina-6-Cfucosídeo. Todos apresentam-se na forma glicosilada e possuem uma hidroxila primária na molécula a qual é necessária para a reação de esterificação/transesterificação utilizando lipase de Candida_antarctica tipo B (CALB) como catalisador. Destes, os que estão em maior quantidade na planta são a isoorientina e a isovitexina_(Li et al., 2011). Suas estruturas químicas com o ponto de funcionalização indicado com uma seta estão ilustradas na Figura 1.

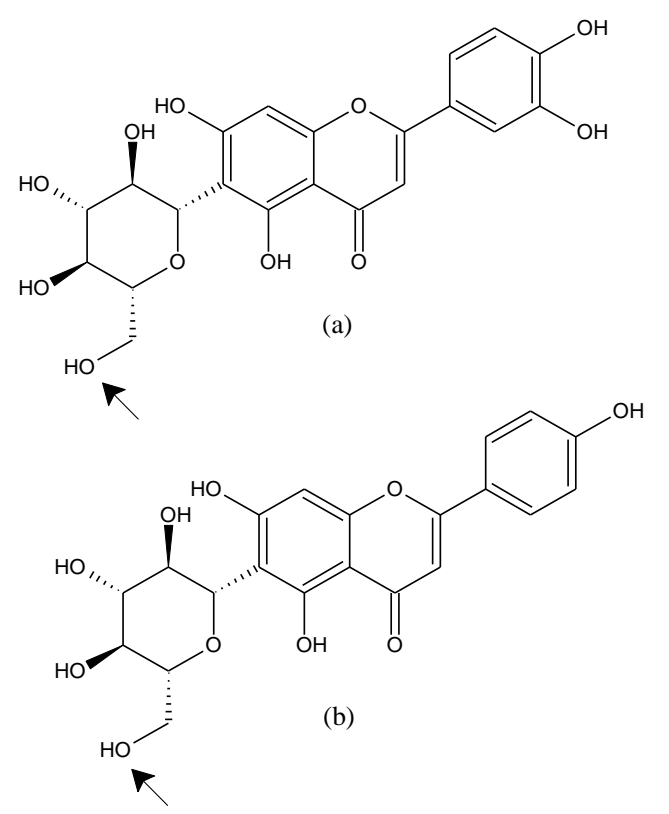

Figura 1 - Estruturas químicas dos flavonoides isoorientina (a) e isovitexina (b) com seus pontos susceptíveis a esterificação utilizando CALB (indicados por uma seta).

A extração de compostos fenólicos a partir de produtos naturais tem atraído, nos últimos anos, o desenvolvimento de pesquisas no intuito de desenvolver processos e otimizar o rendimento da extração (Pinelo et al., 2005). Já que constitui uma das etapas mais importantes no isolamento, identificação e aplicação de compostos fenólicos, os métodos de extração tem sido diversificados desde os métodos tradicionais através do uso de solventes (extração líquidolíquido, sólido-líquido), como métodos não convencionais (extração por fluido supercrítico, extração por ultrassom, extração mecânico-química, extração por micro-ondas, extração com 
infravermelho e extração com líquido pressurizado) (Chua, 2013, Xie_et al., 2011, Liu et al., 2014). Independentemente da técnica de extração, a extração sólido-líquido ocorre em dois estágios: (1) tumefação e hidratação da matriz vegetal e (2) transferência de massa do soluto a partir da matriz vegetal para o solvente por difusão e pressão osmótica (Vinatoru, 2001). Cada método possui vantagens e desvantagens, cuja escolha influencia diretamente o rendimento e a pureza do soluto. As principais variáveis que influenciam no processo são temperatura, razão sólido-líquido, tempo de extração, número de ciclos e concentração e tipo de solvente (Liu et al. 2014, Xie et al., 2011). Em estudo da otimização de extração sólido-líquido de flavonoides totais a partir de Scutellaria_baicalenses, Liu et al. (2014) obtiveram um rendimento de 19,437 $\mathrm{mg}$ flavonoides/g massa seca em condições otimizadas.

Desta forma, este trabalho teve como objetivo avaliar métodos de extração sólidolíquido sobre o rendimento de extração de flavonoides a partir da casca de Passiflora edulis (maracujá amarelo) para posterior esterificação utilizando lipase imobilizada em suportes nanoestruturados.

\section{MATERIAL E MÉTODOS}

\subsection{Matéria-prima}

Foi utilizada como matéria-prima a casca do maracujá seca e pulverizada adquirida comercialmente.

\subsection{Determinação do perfil cromatográfico e quantificação dos flavonoides glicosilados a partir dos extratos hidroetanólicos}

As análises foram conduzidas em cromatógrafo líquido Thermo Fisher Scientific acoplado a um detector UV/PAD e controlado pelo software_ChromQuest. A separação e quantificação foi realizada em coluna Lichrospher RP-18 $(250 \mathrm{~mm}$ x $4 \mathrm{~mm} ; 5 \mu \mathrm{m})$ fornecida pela Merck. As análises foram conduzidas a temperatura ambiente, utilizando como eluente uma mistura acetonitrila/água deionizada (20:80), ajustada com ácido trifluoroacético para pH 3,0. Empregou-se uma vazão de $0,8 \mathrm{~mL} / \mathrm{min}$. As amostras, extratos hidroetanólicos, foram injetadas automaticamente $(20 \mu \mathrm{L})$ e monitoradas a $340 \mathrm{~nm}$. Utilizou-se como flavonóides padrões a isoorientina e a isovitexina grau HPLC (98\%), ambas fornecidas pela Sigma. A curva de calibração foi construída com soluções dos padrões em etanol $60 \%$ (v/v) com concentrações variando de 10 a $40 \mathrm{mg} / \mathrm{L}$. Utilizou-se acetonitrila grau HPLC (TEDIA-Brazil) e os demais solventes foram de grau analítico.

\subsection{Técnicas de extração}

Foram avaliadas as seguintes técnicas de extração: sólido-líquido em regime estático, Soxhlet e ultrassônica. Utilizou-se como solvente solução de etanol 60 \% (v/v) e as condições operacionais foram obtidas a partir da literatura (Ciric et al., 2012 e Liu et al., 2014). O extrato hidroetanólico final de cada tipo de extração, obtido pela junção das frações de cada ciclo, foi diluído para $100 \mathrm{~mL}$ para posterior quantificação do teor de flavonoides.

Extração sólido-líquido: A extração foi conduzida em tubos de centrífuga de $50 \mathrm{~mL}$ mantidos a temperatura ambiente e sem agitação. Pesou-se 1,0 g da amostra e, em seguida, 
adicionou-se $20 \mathrm{~mL}$ do solvente. Após cada ciclo, as amostras foram centrifugadas a $600 \mathrm{~g}$, durante $5 \mathrm{~min}$ a $10^{\circ} \mathrm{C}$, separando-se o extrato e adicionando-se, em seguida, $20 \mathrm{~mL}$ de solvente fresco. O tempo total de extração foi 4,5 h dividido em três ciclos de 1,5 h.

Extração Soxhlet: $1,0 \mathrm{~g}$ de amostra foi adicionada ao extrator, juntamente com $100 \mathrm{~mL}$ do solvente. O processo foi conduzido a $60{ }^{\circ} \mathrm{C}$ durante $2 \mathrm{~h}$ em ciclo único. Após este período as amostras foram centrifugadas a $600 \mathrm{~g}$, por $5 \mathrm{~min}$ a $10^{\circ} \mathrm{C}$.

Extração por Ultrassom: A extração foi conduzida em sonicador UltraCleaner Modelo 1450A. Uma amostra de $2,5 \mathrm{~g}$ do pó da casca de maracujá foram adicionados em bécher, juntamente com $50 \mathrm{~mL}$ de solvente. A operação foi conduzida por 1,0 h dividida em dois ciclos de $0,5 \mathrm{~h}$. Após cada ciclo as amostras foram centrifugadas a $600 \mathrm{~g}$, por 5 min a $10^{\circ} \mathrm{C}$. A operação foi conduzida a $25^{\circ} \mathrm{C}$ e a $50^{\circ} \mathrm{C}$.

As condições operacionais estão sumarizadas na Tabela 1. Todas as operações foram conduzidas em triplicata.

Tabela 1 - Técnicas de extração e condições operacionais

\begin{tabular}{|l|c|c|c|c|}
\hline \multicolumn{1}{|c|}{ Técnica } & $\begin{array}{c}\text { Temperatura } \\
\left({ }^{\mathbf{}} \mathbf{C}\right)\end{array}$ & $\begin{array}{c}\text { Razão sólido/líquido } \\
(\mathbf{g} / \mathbf{m L})\end{array}$ & $\begin{array}{c}\text { Tempo de } \\
\text { Extração (h) }\end{array}$ & $\mathbf{N}^{\mathbf{0}}$ de ciclos \\
\hline $\begin{array}{l}\text { Extração sólido- } \\
\text { líquido }\end{array}$ & 25 & $1 / 20$ & 4,5 & 3 \\
\hline Extração Soxhlet & 60 & $1 / 100$ & 2,0 & 1 \\
\hline $\begin{array}{l}\text { Extração Ultrassom- } \\
\text { assistida }\end{array}$ & 25 & $1 / 20$ & 1,0 & 2 \\
\hline $\begin{array}{l}\text { Extração Ultrassom- } \\
\text { assistida }\end{array}$ & 50 & $1 / 20$ & 1,0 & 2 \\
\hline
\end{tabular}

\section{RESULTADOS}

A Figura 2 mostra o perfil cromatográfico do extrato hidroetanólico obtido a partir da extração dos flavonoides da casca do maracujá.

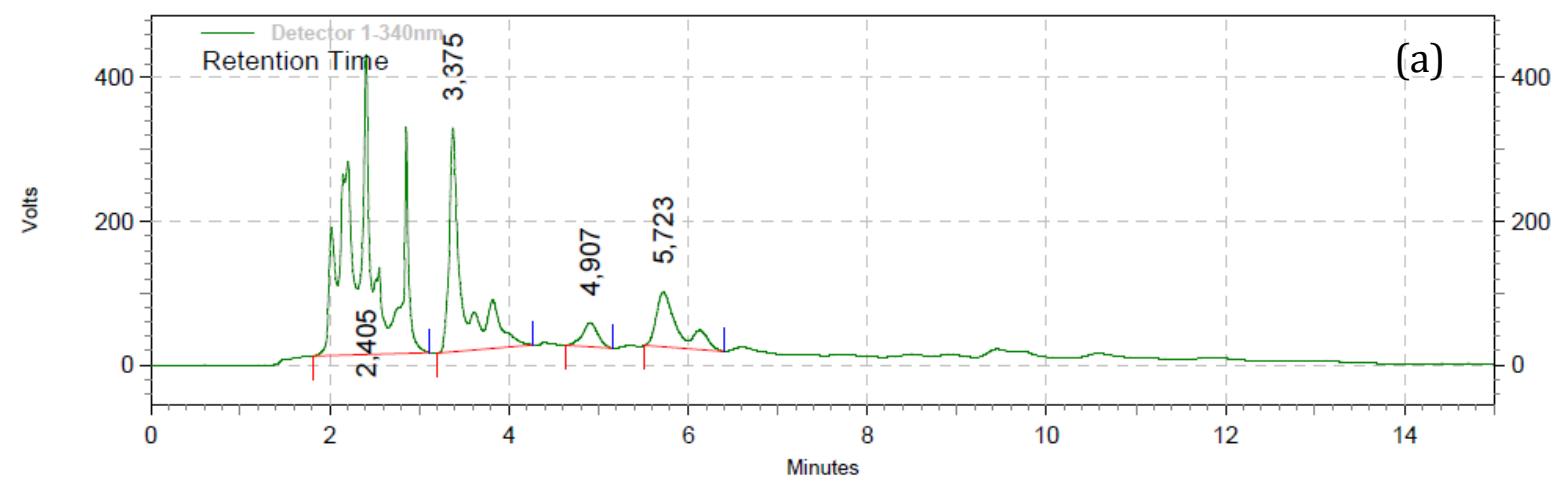



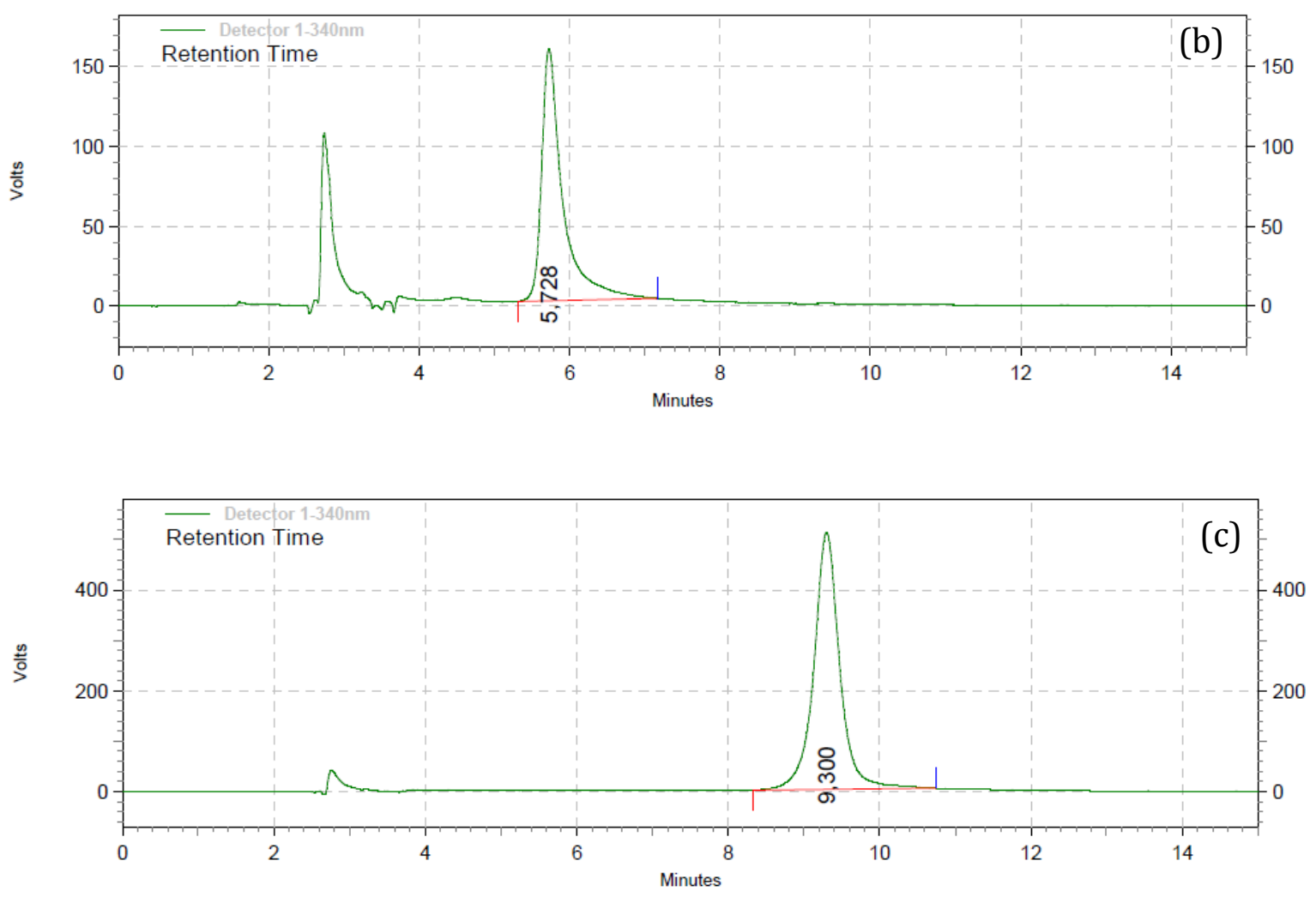

Figura 2- Perfis cromatográficos de (a) extrato hidroetanólico da casca do maracujá, (b) solução padrão de isoorientina e (c) solução padrão de isovitexina

Observou-se a presença do pico característico de isoorientina em 5,723 min (Figura 2(a)) no extrato. Dada a complexidade da amostra, a isovitexina não foi identificada através do método utilizado. Zeraik e Yariwake (2010) obtiveram resultado semelhante ao verificarem que, no extrato hidroetanólico da polpa de Passiflora edulis, a isovitexina não foi o principal pico, bem como, a separação cromatográfica não foi satisfatória.

Os resultados da quantificação de flavonoides nos extratos hidroetanólicos e do teor de flavonoides na casca do maracujá obtidos a partir dos métodos de extração avaliados estão apresentados na Tabela 2.

Tabela 2 - Concentração dos extratos hidroetanólicos e teor de isoorientina na casca do maracujá

\begin{tabular}{|c|c|c|}
\hline Técnica & $\begin{array}{c}\text { Concentração } \\
(\mathrm{mg} / \mathrm{L})\end{array}$ & $\begin{array}{c}\text { Teor (mg_isoorientina/g } \\
\text { casca) }\end{array}$ \\
\hline Sólido-líquido & $4,753 \pm 0,060$ & 0,48 \\
\hline Soxleht $60^{\circ} \mathrm{C}$ & $7,895 \pm 0,355$ & 0,79 \\
\hline Ultrassom $25^{\circ} \mathrm{C}$ & $9,919 \pm 0,166$ & 0,40 \\
\hline Ultrassom $5^{\circ} \mathrm{C}$ & $9,927 \pm 0,257$ & 0,40 \\
\hline
\end{tabular}


A casca de maracujá apresentou maior teor de flavonoides, expresso como_isoorientina, equivalente a $0,79 \mathrm{mg} / \mathrm{g}$ casca seca e concentração de extrato hidroetanólico_de 9,927 \pm 0,257 $\mathrm{mg} / \mathrm{L}$. O teor de flavonoides foi comparativamente equivalente ou maior ao reportado para outras fontes de flavonoides encontradas na literatura, tais como, cebola $(0,71-0,80)$ (Rhodes e Price, 1996), tomate $(0,005$ - 0,03) (Willcox et al., 2003), mel (0,03) (Yao et al., 2003) expressos em termos de $\mathrm{mg}$ de flavonoides/g de matéria-prima. Com relação ao bagaço de canade-açúcar (0,38 mg flavonoides/g bagaço fresco) (Colombo et al., 2006), um resíduo agroindustrial, a casca de maracujá também apresentou maior teor de flavonoides. Estes resultados mostraram que a casca do maracujá constitui uma fonte promissora destes compostos fitoquímicos.

A avaliação dos métodos de extração foi realizada de acordo com o teor de flavonoides (Tabela 2) e da taxa de extração (Figura 2). O método de extração sólido-líquido conduzido à $25^{\circ} \mathrm{C}$, em regime estático e durante 4,5 horas apresentou baixa eficiência, tanto em termos de teor de flavonoides $(0,48 \mathrm{mg} / \mathrm{g})$ como com relação à taxa de extração $(0,1 \mathrm{mg} / \mathrm{g} / \mathrm{h})$. A extração Soxleht realizada à $60^{\circ} \mathrm{C}$, durante 2 horas e razão sólido/líquido de $1 / 100$ apresentou maior teor de flavonoides com relação aos demais métodos (Tabela 2). Já a taxa de extração deste método foi equivalente à extração com ultrassom (Figura 2), em ambas as temperaturas estudadas. A variação da temperatura de $25^{\circ} \mathrm{C}$ para $50{ }^{\circ} \mathrm{C}$ na extração ultrassônica não exerceu influência significativa tanto no teor de flavonoides quanto na taxa de extração, como mostram os resultados na Tabela 2 e Figura 2. Observou-se, portanto, que a extração ultrassônica foi o método mais adequado por apresentar uma taxa de extração equivalente ao método Soxleht, à temperatura ambiente $\left(25^{\circ} \mathrm{C}\right)$ e com menor volume de solvente $(20 \mathrm{~mL}$ de solvente/g de casca).

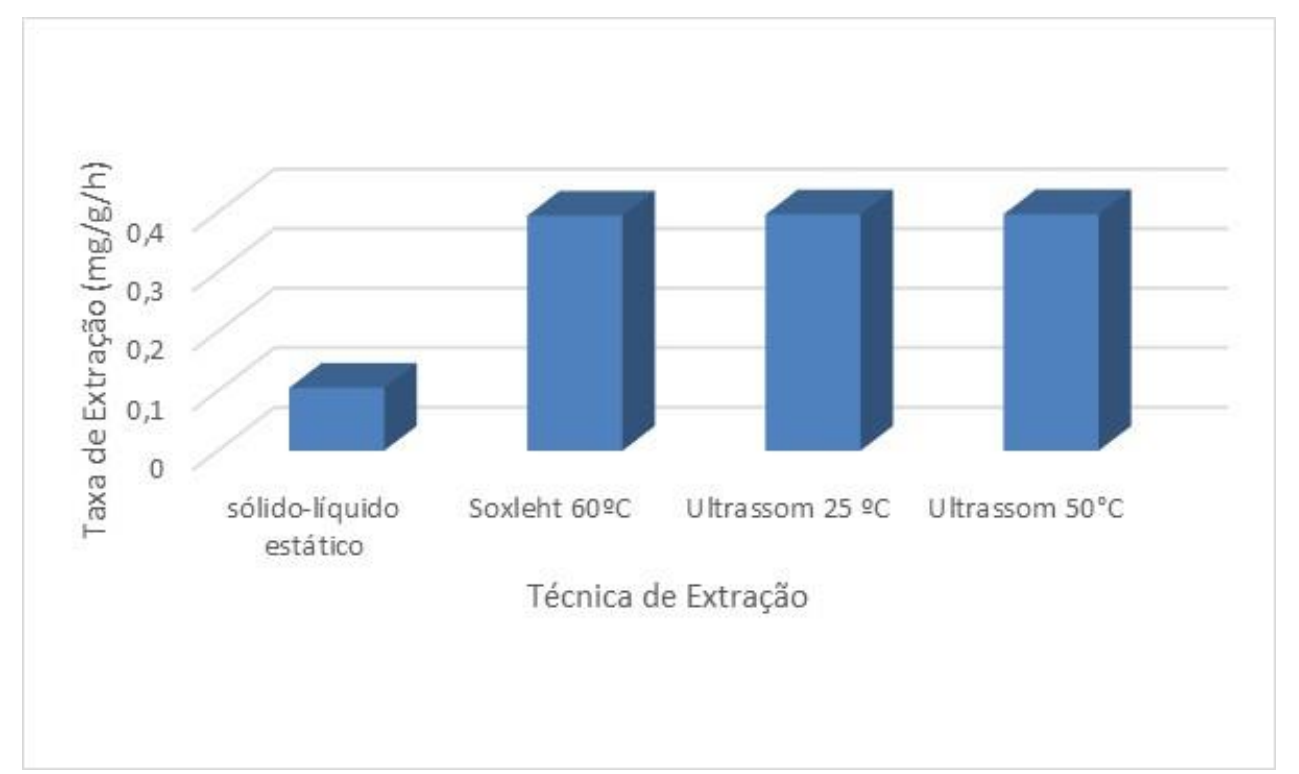

Figura 2 - Taxas de extração em função das técnicas utilizadas

O efeito do aumento da temperatura e do refluxo de solvente na extração Soxleht foram consideravelmente importantes para a operação, pois favoreceram a dissolução e a difusão, fenômenos que governam a transferência de massa de compostos químicos a partir de matrizes 
vegetais nesta operação (Chua, 2013). Entretanto, a literatura aponta como desvantagens deste método o emprego de temperaturas elevadas que podem degradar compostos químicos, bem como, o uso de elevado volume de solvente (Chua, 2013 e Ignat_et al., 2011). Na extração ultrassônica, Chua (2013) atribuiu o aumento da transferência de massa ao fenômeno de cavitação acústica que promove um incremento da área de contato entre o material sólido e o solvente. Este fenômeno possivelmente governou o processo de extração, dentro da faixa de temperatura estudada. Ignat et al. (2011) ressaltaram ainda que a extração ultrassônica consiste em um método simples e mais eficiente que as técnicas convencionais de extração.

\section{4 - CONCLUSÕES}

A casca de $P$. edulis (maracujá amarelo) constituiu uma fonte de flavonoides promissora e de baixo custo, apresentando teor de isoorientina equivalente a $0,79 \mathrm{mg} / \mathrm{g}$ de casca seca.

A técnica de extração ultrassônica foi mais eficiente que as demais técnicas convencionais de extração sólido-líquido, apresentando uma taxa de extração de $0,40 \mathrm{mg} / \mathrm{g} / \mathrm{h}$ à temperatura ambiente e com reduzido volume de solvente $(20 \mathrm{~mL} / \mathrm{g}$ de casca).

\section{5 - REFERÊNCIAS}

AO, L. H.; DATTA, N.; TOMAS-BARBERAN, F. A.; MARTOS, I.; FERRERES, F.; SINGANUSONG, R. Flavonoids, phenolic acids and abscisic acid in Australian and New Zealand Leptospermum honeys, Food Chemistryv, v. 81, p. 159 -168, 2003.

ARCOS, J. A.; HILL, C. G.; OTERO, C. Kinetics of the lipase-catalyzed synthesis of glucose esters in acetone. Biotechnol. Bioeng., v. 73, p. 104-110, 2001.

BENDINI, A.; CERRETANI, L.; PIZZOLANTE, L.; TOSCHI, T. G.; GUZZO, F.; CEOLDO, S.; MARCONI, A. M.; ANDREETTA, F.; LEVI, M. Phenol content related to antioxidant and antimicrobial activities of Passiflora spp. extracts. Eur. Food Res. Technol., v.223, p. 102-109, 2006.

CHUA, L. S., A review on plant-based rutin extraction methods and its pharmacological activities, Journal of Ethnopharmacology, v. 150, pp. 805 - 817, 2013.

CIRIC, A., PROSEN, H., JELIKIC-STANKOV, M., DURDEVIC, P. Evaluation of matrix effect in determination of some bioflavonoids in food samples by LC-MS/MS method, Talanta, v. 99, $780-790,2012$.

COLOMBO, R., LANÇAS, F. M., YARIWAKE, J. H., Determination of flavonoids in cultivated sugarcane Leaves, bagasse, juice and in transgenic sugarcane by liquid chromatography-UV detection, Journal of Chromatography A, v. 1103, p. 118 - 124, 2006.

IGNAT, I., VOLF, I., POPA, V. I., A critical review of methods for characterisation of polyphenolic compounds in fruits and vegetables, Food Chemistry, v. 126, p. 1821-1835, 2011. 
LI, H.; ZHOU, P.; YANG, Q.; SHEN, Y.; DENG, J.; LI, L.; ZHAO, D. Comparative studies on anxiolytic activities and flavonoid compositions of Passiflora edulis edulis and Passiflora edulis flavicarpa. J. Ethnol., v. 133, p. 1085-1090, 2011.

LIU, Y., HONGWU, W., CAI, X. Optimization of the extraction of total flavonoids from Scutellaria baicalensis Georgi using the response surface methodology, Journal of Food Science Technology, 2014

OLIVEIRA, L. F.; NASCIMENTO, M. R. F.; BORGES, S. V.; RIBEIRO, P. C. N.; RUBACK, V. R. Aproveitamento alternativo da casca do maracujá amarelo (Passiflora edulis f. flavicarpa deg.) para a produção de doce em calda, Cien. Tecnol. Alim., v. 22, p. 259-262, 2002.

PASSICOS, E.; SANTARELLI, X.; COULON, D. Regioselective acylation of flavonoids catalyzed by immobilized Candida antarctica lipase under reduced pressure. Biotechnol. Lett., v. 26, p. 1073-1076, 2004.

PINELO, M., RUBILAR, M., JEREZ, M., SINEIRO, J., NUNEZ, M. J., Effect of solvent, temperature, and solvent-to-solid ratio on the total phenolic content and antiradical activity of extracts from different components of grape pomace. J. Agric. Food Chem, v. 53, p. 2111 2117, 2005.

RHODES, M. J. C., PRICE, K.R., Analytical problems in the study of flavonoid compounds in onions, Food Chem., v. 57, p. 113, 1996.

RUDNICKI, M.; DE OLIVEIRA, M. R.; PEREIRA, T. V.; REGINATTO, F. H.; DALPIZZOL, F.; MOREIRA, J. C. F. Antioxidant and antiglycation properties of Passifloraalata and Passiflora edulis extracts. Food Chem., v. 100, 719-724, 2007.

SHARMA, S., KANWAR, S. S. Organic Solvent tolerant lipases and applications, The scientific world jornal, v. 2014, 2014.

VINATORU, M., An overview of the ultrassonically assisted extraction of bioactive principles from herbs, Ultrasonics Sonochemistry, v. 8, p. 303, 2001.

WANG, A.; ZHANG, F.; HUANG, Y.; LI, H.; WANG, Q.; ZHAOWU, Z.; XIE, T. New progress in biocatalysis and biotranformation of flavonoids. J. Med. Plant Res., v. 4, p. 847856, 2010.

WILLCOX J.K., CATIGNANI G.L., LAZARUS S., Tomatoes and cardiovascular health. Crit. Ver. Food Sci. Nutr., v. 43, p. 1-18, 2003.

XIE, J., SHI, L., ZHU, X., WANG, P., ZHAO, Y., SU, W. Mechanochemical-assisted eficiente extraction of rutin from Hibiscus mutabilis L., Innovative Food Science and Emerging Technologies, v. 12, p. 146 - 152, 2011. 